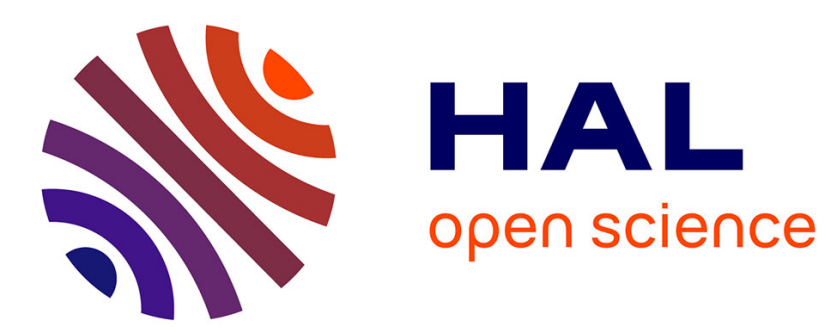

\title{
Mirrored Sampling in Evolution Strategies With Weighted Recombination
}

\author{
Anne Auger, Dimo Brockhoff, Nikolaus Hansen
}

\section{To cite this version:}

Anne Auger, Dimo Brockhoff, Nikolaus Hansen. Mirrored Sampling in Evolution Strategies With Weighted Recombination. Genetic and Evolutionary Computation Conference (GECCO 2011), SIGEVO, Jul 2011, Dublin, Ireland. pp.861-868, 10.1145/2001576.2001694 inria-00612522

\section{HAL Id: inria-00612522 \\ https://hal.inria.fr/inria-00612522}

Submitted on 29 Jul 2011

HAL is a multi-disciplinary open access archive for the deposit and dissemination of scientific research documents, whether they are published or not. The documents may come from teaching and research institutions in France or abroad, or from public or private research centers.
L'archive ouverte pluridisciplinaire HAL, est destinée au dépôt et à la diffusion de documents scientifiques de niveau recherche, publiés ou non, émanant des établissements d'enseignement et de recherche français ou étrangers, des laboratoires publics ou privés. 


\title{
Mirrored Sampling in Evolution Strategies With Weighted Recombination
}

\author{
Anne Auger \\ TAO Team, INRIA Saclay-Île-de-France \\ LRI, University Paris Sud \\ 91405 Orsay Cedex, France \\ firstname.lastname@inria.fr \\ Dimo Brockhoff \\ Sysmo Team \\ Nikolaus Hansen \\ LIX, Ecole Polytechnique \\ 91128 Palaiseau Cedex, France \\ TAO Team, INRIA Saclay-Île-de-France \\ LRI, University Paris Sud \\ 91405 Orsay Cedex, France \\ brockho@lix.polytechnique.fr firstname.lastname@inria.fr
}

\begin{abstract}
This paper introduces mirrored sampling into evolution strategies (ESs) with weighted multi-recombination. Two further heuristics are introduced: pairwise selection selects at most one of two mirrored vectors in order to avoid a bias due to recombination. Selective mirroring only mirrors the worst solutions of the population. Convergence rates on the sphere function are derived that also yield upper bounds for the convergence rate on any spherical function. The optimal fraction of offspring to be mirrored is regardless of pairwise selection one without selective mirroring and about $19 \%$ with selective mirroring, where the convergence rate reaches a value of 0.390 . This is an improvement of $56 \%$ compared to the best known convergence rate of 0.25 with positive recombination weights.
\end{abstract}

\section{Categories and Subject Descriptors}

G.1.6 [Numerical Analysis]: Optimization-global optimization, unconstrained optimization; F.2.1 [Analysis of Algorithms and Problem Complexity]: Numerical Algorithms and Problems

\section{General Terms}

Algorithms, Theory

\section{INTRODUCTION}

Derandomization of random numbers is a general technique where independent samples are replaced by dependent ones. Recent studies showed, for example, how derandomization via mirrored sampling can improve $(1, \lambda)$ - and $(1+\lambda)$-ESs $[6,3]$. Instead of generating $\lambda$ independent and identically distributed (i.i.d.) search points in iteration $k$ as $\boldsymbol{X}_{k}+\sigma_{k} \mathcal{N}^{i}$ where $\boldsymbol{X}_{k}$ is the current search point, $\sigma_{k}$ the current step-size, and $\mathcal{N}^{i}$ a random sample from a multivariate normal distribution, the $(1+\lambda)$-ES with mirrored sampling always pairs samples one by one and produces $\lambda / 2$ independent search points $\boldsymbol{X}_{k}+\sigma_{k} \mathcal{N}^{i}$ and $\lambda / 2$ dependent

(C)ACM, 2011. This is the authors' version of the work. It is posted here by permission of ACM for your personal use. Not for redistribution. The definitive version was published at GECCO'11, July 12-16, 2011, Dublin, Ireland. http://doi.acm.org/10.1145/2001576.2001694 ones as $\boldsymbol{X}_{k}-\sigma_{k} \mathcal{N}^{i}(1 \leq i \leq \lambda / 2)$. In the end, the best out of these $\lambda$ search points is used as next search point $\boldsymbol{X}_{k+1}$ in the $(1, \lambda)$-ES (and in the $(1+\lambda)$-ES the best out of the $\lambda$ new and the old $\boldsymbol{X}_{k}$ ). Several ES variants using mirrored mutations showed noticeable improvements over their unmirrored counterparts - not only in theoretical investigations on simple test functions such as the sphere function, but also in exhaustive experiments within the $\mathrm{COCO} / \mathrm{BBOB}$ framework $[6,3]$. Up to now, the results were restricted to single-parent $(1+\lambda)$-ESs though the idea is, in principle, applicable in a straight-forward manner to population-based ESs such as the $\left(\mu / \mu_{\mathrm{w}}, \lambda\right)$-ES where the $\mu$ best out of the $\lambda$ offspring are used to compute the new search point $\boldsymbol{X}_{k+1}$ via weighted recombination. However, the direct application of mirrored mutations in population-based ESs, as for example proposed in a more general way by Teytaud et al. [12], results in an undesired bias on the step-size, as was argued already in [6].

The purpose of this paper is to introduce mirrored mutations into ESs with weighted recombination without introducing a bias on the length of the recombined step. The main idea hereby is pairwise selection that allows only the better solution of a mirrored/unmirrored solution pair to possibly contribute to the weighted recombination. In detail, the contributions of this paper are

- the introduction of several ES variants that combine mirrored mutations and weighted recombination without a bias on the recombined step,

- a theoretical investigation of the algorithms' convergence rates (in finite and infinite dimension) on spherical functions,

- the computation of optimal recombination weights, and

- an experimental comparison of convergence rates with only positive recombination weights, in particular evaluating the impact of mirrored mutations.

The paper is organized as follows. After introducing the baseline $\left(\mu / \mu_{\mathrm{w}}, \lambda\right)$-ES, Sec. 2 explains in detail how mirrored mutations can be introduced in this algorithm. Section 3 theoretically investigates the convergence rate of three variants in finite and infinite dimension. Section 4 presents a comparison of the algorithms based on the numerical estimation of their convergence rates on the sphere function. Section 5 summarizes and concludes the paper.

Notations. For a (random) vector $\boldsymbol{x} \in \mathbb{R}^{n},[\boldsymbol{x}]_{1}$ will denote its first coordinate. The vector $(1,0, \ldots, 0)$ will be denoted $\boldsymbol{e}_{1}$. A random vector following a multivariate normal distribution with mean vector zero and covariance matrix identity will be called standard multivariate normal distribution. 


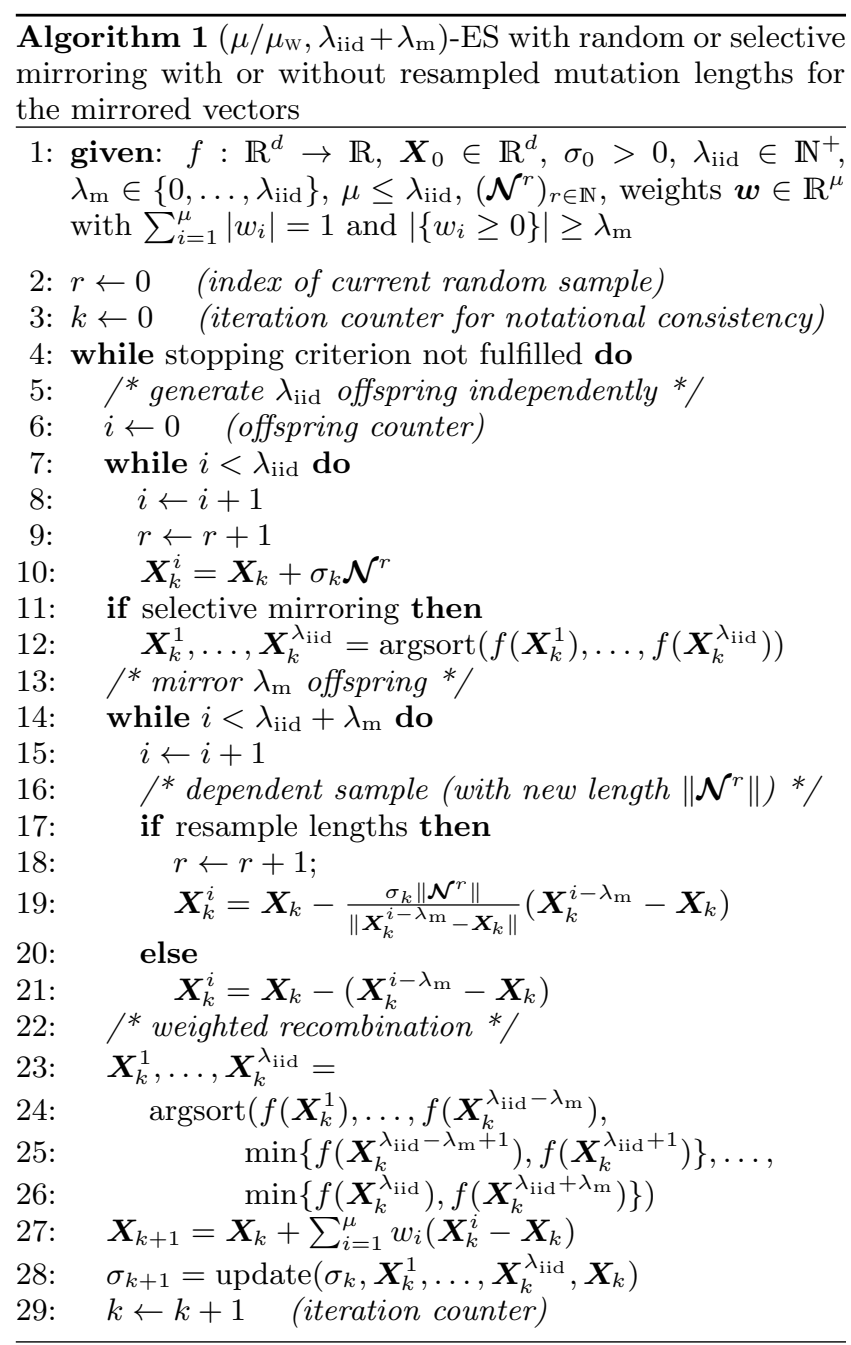

\section{MIRRORING AND WEIGHTED RECOM- BINATION}

\subsection{The Standard $\left(\mu / \mu_{\mathrm{w}}, \lambda\right)$-ES}

As our baseline algorithm, we briefly recapitulate the standard $\left(\mu / \mu_{\mathrm{w}}, \lambda\right)$-ES with weighted recombination and show its pseudo code in Algorithm 1, where $\lambda_{\mathrm{m}}=0$ and therefore $\lambda=\lambda_{\text {iid }}$. Weighted recombination generalizes intermediate multi-recombination (where all weights are equal), has been studied in $[10,7,2]$, and must be nowadays regarded as standard in ESs. Given a starting point $\boldsymbol{X}_{0} \in \mathbb{R}^{d}$, an initial step-size $\sigma_{0}>0$, a population size $\lambda \in \mathbb{N}^{+}$, and weights $\boldsymbol{w} \in \mathbb{R}^{\mu}$ with $\sum_{i=1}^{\mu}\left|w_{i}\right|=1$ for a chosen $1 \leq \mu \leq \lambda$, the $\left(\mu / \mu_{\mathrm{w}}, \lambda\right)$-ES generates at iteration $k \lambda$ independent search points from a multivariate normal distribution with mean $\boldsymbol{X}_{k}$ and variance $\sigma_{k}^{2}$ and recombines the best $\mu$ of them in terms of a weighted sum to become the new mean $\boldsymbol{X}_{k+1}$ of the next iteration (in case of negative weights, the steps must be recombined, see line 27 in Algorithm 1).

Typically, $\mu$ is chosen as $\lfloor\lambda / 2\rfloor$ and $w_{i}=\ln \left(\frac{\lambda+1}{2}\right)-\ln (i)>$ 0 in the scope of the CMA-ES [7]. As update rule for the step-size $\sigma_{k}$ in the $\left(\mu / \mu_{\mathrm{W}}, \lambda\right)$-ES, several techniques such as self-adaptation [11] or cumulative step-size adaptation [9] are available. Of particular theoretical interest is the scale-

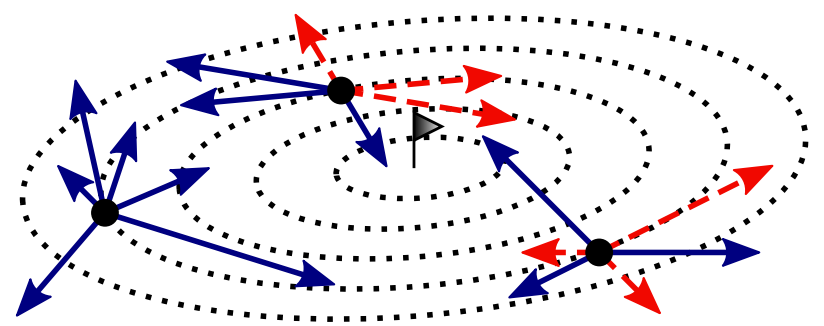

Figure 1: Illustration of i.i.d. mutations (left) and mirrored mutations (middle) and mirrored mutations with resampled lengths (right). Dashed arrows depict the mirrored samples. Dotted lines connect points with equal function value.

invariant (constant) step-size $\sigma_{k}=\sigma\left\|\boldsymbol{X}_{k}\right\|$ which depends on the distance to the optimum assumed WLOG in zero and which allows to prove bounds on the convergence rate of evolution strategies with any adaptive step-size update, see Sec. 3.

\subsection{The Mirroring Idea}

Derandomized mutations [12] and more recently mirrored mutations $[6,3]$ have been proposed to replace the independent mutations in evolution strategies by dependent ones in order to reduce the probability of "unlucky events"resulting in an increase in the convergence speed of the algorithms. Instead of sampling all $\lambda$ offspring i.i.d., an algorithm with mirrored mutations samples only $\lceil\lambda / 2\rceil$ i.i.d. offspring as $\boldsymbol{X}_{k}^{i}=\boldsymbol{X}_{k}+\sigma_{k} \mathcal{N}^{i}(1 \leq i \leq\lceil\lambda / 2\rceil)$ and up to $\lfloor\lambda / 2\rfloor$ further offspring depending on the already drawn samples as $\boldsymbol{X}_{k}^{i}=\boldsymbol{X}_{k}-\sigma_{k} \mathcal{N}^{i-\lceil\lambda / 2\rceil}$ (for $\lceil\lambda / 2\rceil+1 \leq i \leq \lambda$ ), see Fig. 1, left versus middle.

In evolution strategies with weighted recombination and cumulative step-size adaption, mirrored mutations cause a bias towards smaller step-sizes [6, Fig. 4], see Fig 2. The bias can cause premature convergence of the algorithm. ${ }^{1}$ The reason for the bias is that if both samples $\boldsymbol{X}_{k}+\sigma_{k} \mathcal{N}^{i}$ and $\boldsymbol{X}_{k}-\sigma_{k} \mathcal{N}^{i}$ are considered within weighted recombination, they partly cancel each other out and the realized shift of $\boldsymbol{X}_{k}$ will be smaller than with independent mutations. Consequently, derandomized step-size control like cumulative stepsize adaptation [9] will cause the step-size to shrink.

In this paper, we therefore introduce pairwise selection which prevents this bias: unmirrored and mirrored offspring are paired two-by-two and only the better among the unmirrored sample $\boldsymbol{X}_{k}+\sigma_{k} \mathcal{N}^{i}$ and its mirrored counterpart $\boldsymbol{X}_{k}-\sigma_{k} \mathcal{N}^{i}$ is used within the weighted recombination but never both. Here, we introduce a new notation: the number of independent offspring per iteration is denoted by $\lambda_{\text {iid }}$ and the number of mirrored offspring per iteration is denoted by $\lambda_{\mathrm{m}}$, where each iteration $\lambda=\lambda_{\text {iid }}+\lambda_{\mathrm{m}}$ solutions are evaluated on $f$. As a result $0 \leq \lambda_{\mathrm{m}} \leq \lambda_{\text {iid }}$ which results in the standard $\left(\mu / \mu_{\mathrm{W}}, \lambda\right)$-ES in case $\lambda_{\mathrm{m}}=0$. We denote the new algorithm $\left(\mu / \mu_{\mathrm{w}}, \lambda_{\mathrm{iid}}+\lambda_{\mathrm{m}}\right)$-ES.

Note that the idea of sequential mirroring of $[6,3]$, i.e., stopping the generation of new offspring as soon as a better solution than the parent is found, is not applied here. With

\footnotetext{
${ }^{1}$ Mutative self-adaptation has no such bias, but suffers in combination with weighted recombination from a far too small control target step-size and can achieve close to optimal step-sizes only with a peculiar parameter tuning.
} 


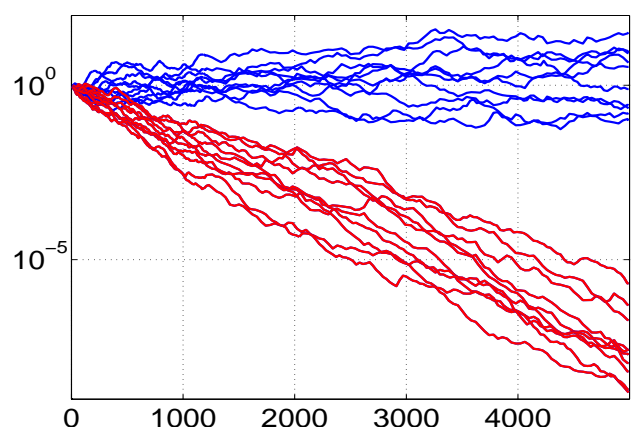

Figure 2: Illustration of the bias towards smaller step-sizes under random selection introduced by recombination of mirrored vectors in the CMA-ES. Shown is the step-size $\sigma$ versus the number of function evaluations of 20 runs on a purely random fitness function in dimension 10. The upper ten graphs show the $\left(5 / 5_{\mathbf{w}}, 10\right)$-CMA-ES revealing a random walk on $\log (\sigma)$. The lower ten graphs show the $\left(5 / 5_{\mathrm{w}}, 5+5_{\mathrm{m}}\right)$-CMA-ES without pairwise selection of mirrored samples.

recombination, the meaning of a comparison with "the parent" is not unique and additional algorithm design decisions were necessary ${ }^{2}$. Instead, selective mirroring is introduced.

\subsection{Random Versus Selective Mirroring}

We consider two variants of the $\left(\mu / \mu_{\mathrm{w}}, \lambda_{\text {iid }}+\lambda_{\mathrm{m}}\right)-\mathrm{ES}^{3}$ that differ in the choice of mirrored offspring. The $\left(\mu / \mu_{\mathrm{W}}, \lambda_{\mathrm{iid}}+\right.$ $\left.\lambda_{\mathrm{m}}^{\mathrm{rand}}\right)$-ES, where $\lambda_{\mathrm{m}}$ randomly chosen offspring are mirrored, and the $\left(\mu / \mu_{\mathrm{W}}, \lambda_{\mathrm{iid}}+\lambda_{\mathrm{m}}^{\mathrm{sel}}\right)$-ES with selective mirroring, where only the $\lambda_{\mathrm{m}}$ worst offspring are selected for mirroring.

The reason behind the latter variant of selecting the worst offspring for mirroring is the following: in particular on fitness functions with convex sublevel sets ${ }^{4}$ we do not expect the best of $\lambda_{\text {iid }}$ offspring to improve by mirroring. For an offspring that is better than the current search point $\boldsymbol{X}_{k}$, the mirroring would always result in a worse solution since never both an independently drawn solution and its mirrored counterpart can be better than the parent in case of convex sublevel sets [3, Proposition 2].

Regarding the comparison of random and selective mirroring, two questions arise: (i) how much faster can an ES become with selective mirroring and (ii) what is the optimal choice for the number $\lambda_{\mathrm{m}}$ of mirrored offspring. Both questions will be answered in the following by theoretical investigations of the algorithms' convergence rates.

\footnotetext{
${ }^{2}$ The super-parent and distribution mean $\boldsymbol{X}_{k}$, resulting from the weighted recombination, is not directly comparable to the offspring, because depending on $d, \mu$ and $\lambda$ with a large probability all i.i.d. sampled offspring might be worse. However, a feasible heuristic could be to compare with the best offspring from the last iteration.

${ }^{3}$ Adaptive variants with a variable number of mirrored offspring that depends on the observed fitness function values have also been considered but are not included here.

${ }^{4}$ The sublevel set $S_{l}$ contains all points in search space with a fitness value of at most $l: S_{l}=\left\{x \in \mathbb{R}^{d} \mid f(x) \leq l\right\}$.
}

\subsection{Resampled Mirrored Vector Lengths}

Within the $\left(\mu / \mu_{\mathrm{W}}, \lambda\right)$-ES, solutions that happen to originate from a comparatively long step tend to be worse than average. Therefore, the solutions chosen by selective mirroring are biased towards long mutation steps and their mirrors tend to be bad solely because they originate from a long mirrored step (still they tend to be better than the original $\lambda_{\text {iid }}$ samples). Hence, we consider a variant of mirroring where the lengths of the mirrored vectors $\sigma_{k} \mathcal{N}^{r}$ are i.i.d. resampled, i.e., where $\boldsymbol{X}_{k+1}=\boldsymbol{X}_{k}-\sigma_{k} \mathcal{N}^{r}$ is replaced by $\boldsymbol{X}_{k+1}=\boldsymbol{X}_{k}-\sigma_{k} \frac{\left\|\mathcal{N}^{r+1}\right\|}{\left\|\mathcal{N}^{r}\right\|} \mathcal{N}^{r}$ with $\left\|\mathcal{N}^{r+1}\right\|$ the newly sampled length of the mirrored vector, cp. Fig. 1, right.

We refer to this last variant as $\left(\mu / \mu_{\mathrm{w}}, \lambda_{\text {iid }}+\lambda_{\mathrm{m}}^{\mathrm{sel}}\right)$-ES with resampled (mutation) lengths. Algorithm 1 shows the pseudo code of all variants with random/selective mirroring and with/without resampled lengths of the mirrored offspring. Theoretical results in the next section will not only show how much improvement in the convergence rate can be gained by the resampled lengths but also that the variants with and without resampled lengths are the same if the dimension goes to infinity.

\subsection{Algorithm Parameters}

The algorithms we have described involve several parameters, the number $\lambda_{\text {iid }}$ of independent samples, the number $\lambda_{\mathrm{m}}$ of mirrored offspring, the number $\mu$ of offspring to be recombined, the weights $\boldsymbol{w}$ for recombination, and the step-size $\sigma_{k}$. The convergence rates depend on the choice of these parameters. In the sequel, we investigate upper bounds for the convergence rate on spherical functions by investigating the optimal choice of the different parameters, given $\sum\left|w_{i}\right|=1$.

\section{CONVERGENCE RATE UPPER BOUNDS ON SPHERICAL FUNCTIONS}

In order to find optimal settings for the different parameters, we investigate convergence rates on spherical functions having WLOG the optimum in zero, i.e., $g(\|x\|), g \in \mathcal{M}$ where $\mathcal{M}$ denotes the set of functions $g: \mathbb{R} \mapsto \mathbb{R}$ that are strictly increasing. Convergence rates depend on the step-size adaptation chosen. We study the case of the scaleinvariant constant step-size where $\sigma_{k}=\sigma\left\|\boldsymbol{X}_{k}\right\|$, that we refer to as scale-invariant ES (however, as most ESs are scale invariant, the name is somewhat abusive). For an optimal choice of the constant $\sigma$, the scale-invariant ES gives the upper bound for convergence rates achievable by any strategy with step-size adaptation on spherical functions (see below). This case is of great relevance, because also practical stepsize control mechanisms can achieve close-to-optimal stepsizes on spherical functions. For different algorithm variants with scale-invariant step-size, we prove linear convergence in expectation in the following sense: there exists a $\mathrm{CR} \in \mathbb{R}$ such that for all $k, k_{0} \in \mathbb{N}$ with $k>k_{0}$

$$
\frac{1}{\Lambda} \frac{1}{k-k_{0}} E\left[-\ln \frac{\left\|\boldsymbol{X}_{k}\right\|}{\left\|\boldsymbol{X}_{k_{0}}\right\|}\right]=\mathrm{CR},
$$

where $\Lambda$ is the number of evaluations per iteration introduced to define the convergence rate per function evaluation. The constant $\mathrm{CR}^{5}$ is the convergence rate of the algorithm

\footnotetext{
${ }^{5}$ Convergence takes place if and only if $\mathrm{CR}>0$, and for non-elitist ESs only numerical integration of CR (expressed
} 
and depends on the dimension $d$ and the parameters $\sigma, \lambda_{\text {iid }}$, $\lambda_{\mathrm{m}}, \mu$ and $\boldsymbol{w}$, see Sections 2.1 and 2.5 and Algorithm 1 . The convergence rate defined in (1) is compatible with almost sure convergence [4]. Hence, we will prove that with scale-invariant step-size, almost surely

$$
-\frac{1}{\Lambda} \frac{1}{k-k_{0}} \ln \frac{\left\|\boldsymbol{X}_{k}\right\|}{\left\|\boldsymbol{X}_{k_{0}}\right\|} \underset{k \rightarrow \infty}{\longrightarrow} \mathrm{CR}
$$

More loosely we may say that $\left\|\boldsymbol{X}_{k+1}\right\| \approx \exp (-\Lambda \mathrm{CR})\left\|\boldsymbol{X}_{k}\right\|$.

\subsection{The $\left(\mu / \mu_{\mathrm{w}}, \lambda\right)$-ES}

To serve as a baseline algorithm for a later comparison with algorithms using mirrored mutations, we first investigate the convergence rate of the scale-invariant version of the standard $\left(\mu / \mu_{\mathrm{w}}, \lambda\right)$-ES (see Algorithm 1 with $\lambda_{\mathrm{m}}=0$ ).

\subsubsection{Finite Dimension Results}

At each step, $\lambda$ independent vectors following a standard multivariate normal distribution $\mathcal{N}^{i}$ are sampled to create the offspring $\boldsymbol{X}_{k}^{i}=\boldsymbol{X}_{k}+\sigma\left\|\boldsymbol{X}_{k}\right\| \mathcal{N}^{i}$. The offspring are ranked according to their fitness function value. We denote $\left(\boldsymbol{Z}_{1: \lambda}, \ldots, \boldsymbol{Z}_{\lambda: \lambda}\right)$ the sorted vector of multivariate normal distributions, such that the best offspring equals $\boldsymbol{X}_{k}+$ $\sigma\left\|\boldsymbol{X}_{k}\right\| \boldsymbol{Z}_{1: \lambda}$, the second best $\boldsymbol{X}_{k}+\sigma\left\|\boldsymbol{X}_{k}\right\| \boldsymbol{Z}_{2: \lambda}$, etc. The distribution of $\left(\boldsymbol{Z}_{1: \lambda}, \ldots, \boldsymbol{Z}_{\lambda: \lambda}\right)$ depends a priori on $\boldsymbol{X}_{k}$. However, in the scale-invariant step-size case on spherical functions the distribution is independent of $\boldsymbol{X}_{k}$ and is determined by ranking of $\left\|\boldsymbol{e}_{1}+\sigma \mathcal{N}^{i}\right\|$ for $i=1 \ldots \lambda$, that can be simplified to ranking $2\left[\mathcal{N}^{i}\right]_{1}+\sigma\left\|\mathcal{N}^{i}\right\|^{2}$. These results are stated in the following lemma.

LEMMA 1. For the scale-invariant $\left(\mu / \mu_{\mathrm{w}}, \lambda\right)$-ES minimizing spherical functions, the probability distribution of the vector $\left(\boldsymbol{Z}_{1: \lambda}, \ldots \boldsymbol{Z}_{\lambda: \lambda}\right)$ is independent of $\boldsymbol{X}_{k}$ and equals

$$
\left(\boldsymbol{Z}_{1: \lambda}, \ldots, \boldsymbol{Z}_{\lambda: \lambda}\right)=\operatorname{argsort}\left\{h_{\sigma}\left(\mathcal{N}^{1}\right), \ldots, h_{\sigma}\left(\mathcal{N}^{\lambda}\right)\right\}
$$

where $h_{\sigma}(\boldsymbol{x})=2[\boldsymbol{x}]_{1}+\sigma\|\boldsymbol{x}\|^{2}$ and $\left(\mathcal{N}^{i}\right)_{1 \leq i \leq \lambda}$ are $\lambda$ independent standard multivariate normal distribution.

Proof. At iteration $k$, starting from $\boldsymbol{X}_{k}$, the distribution of the selected $\mathcal{N}^{i}$ is determined by the ranking of

$$
\left(\left\|\boldsymbol{X}_{k}+\sigma\right\| \boldsymbol{X}_{k}\left\|\mathcal{N}^{i}\right\|\right)_{1 \leq i \leq \lambda} .
$$

Normalizing by $\left\|\boldsymbol{X}_{k}\right\|$ will not change the ranking such that the distribution is determined by the ranking of

$$
\left\|\frac{\boldsymbol{X}_{k}}{\left\|\boldsymbol{X}_{k}\right\|}+\sigma \mathcal{N}^{i}\right\| \text { for } 1 \leq i \leq \lambda .
$$

However, since the distribution of $\mathcal{N}^{i}$ is spherical, the distribution of the selected $\mathcal{N}^{i}$ will be the same if we start from any vector with unit norm (like $\frac{\boldsymbol{X}_{k}}{\left\|\boldsymbol{X}_{k}\right\|}$ ), so WLOG the distribution will be determined by ranking $\left\|\boldsymbol{e}_{1}+\sigma \mathcal{N}^{i}\right\|$ for $1 \leq$ $i \leq \lambda$ or since composing by $g(x)=x^{2}$ will not change the ranking

$$
\left\|\boldsymbol{e}_{1}+\sigma \mathcal{N}^{i}\right\|^{2} \text { for } 1 \leq i \leq \lambda
$$

as an expectation of some continuous random variables and thus as an integral) can reveal the sign of CR. In contrast to our results, "classical" progress-rate derivations $[1,2,5]$ only approximate the actual strategy behavior and the result of the approximation can be comparatively poor for small values of $d / \lambda$. Consequently, the classical progress-rate $\varphi$ might be negative even when de facto convergence takes place, or vice versa [4].
We develop $\left\|\boldsymbol{e}_{1}+\sigma \mathcal{N}^{i}\right\|^{2}$ and obtain $1+2 \sigma\left[\mathcal{N}^{i}\right]_{1}+\sigma^{2}\left\|\mathcal{N}^{i}\right\|^{2}$. Ranking will not be affected if we subtract 1 and divide by $\sigma$ such that the distribution of the selected $\mathcal{N}^{i}$ is determined by the ranking with respect to $h_{\sigma}\left(\mathcal{N}^{i}\right)$.

In the $\left(\mu / \mu_{\mathrm{w}}, \lambda\right)$-ES, the $\mu$ best offspring $\boldsymbol{X}_{k}+\sigma\left\|\boldsymbol{X}_{k}\right\| \boldsymbol{Z}_{i: \lambda}$ for $i=1, \ldots, \mu$ are recombined into the new parent $\boldsymbol{X}_{k+1}=$ $\boldsymbol{X}_{k}+\sigma\left\|\boldsymbol{X}_{k}\right\| \sum_{i=1}^{\mu} w_{i} \boldsymbol{Z}_{i: \lambda}$ where $\left(w_{1}, \ldots, w_{\mu}\right) \in \mathbb{R}^{\mu}$ and $\sum_{i=1}^{\mu}\left|w_{i}\right|=1$. The next theorem gives the expression of the convergence rate associated to the $\left(\mu / \mu_{\mathrm{w}}, \lambda\right)$-ES with scaleinvariant step-size as a function of $\sigma$ and $\boldsymbol{w}=\left(w_{1}, \ldots, w_{\mu}\right)$.

THEOREM 1. For the $\left(\mu / \mu_{\mathrm{w}}, \lambda\right)$-ES with scale-invariant step-size on $g(\|x\|), g \in \mathcal{M},(1)$ and (2) hold and the convergence rate equals

$\mathrm{CR}(\sigma, \boldsymbol{w})=-\frac{1}{2 \lambda} E \ln \left(1+2 \sum_{i=1}^{\mu} \sigma w_{i}\left[\boldsymbol{Z}_{i: \lambda}\right]_{1}+\left\|\sum_{i=1}^{\mu} \sigma w_{i} \boldsymbol{Z}_{i: \lambda}\right\|^{2}\right)$

where $w_{i} \in \mathbb{R}$ and $\sum_{i=1}^{\mu}\left|w_{i}\right|=1$.

ProOF. We start from

$$
\left\|\boldsymbol{X}_{k+1}\right\|=\left\|\boldsymbol{X}_{k}+\sigma\right\| \boldsymbol{X}_{k}\left\|\sum_{i=1}^{\mu} w_{i} \boldsymbol{Z}_{i: \lambda}\right\|
$$

that we normalize by $\left\|\boldsymbol{X}_{k}\right\|$ and take the logarithm

$$
\ln \frac{\left\|\boldsymbol{X}_{k+1}\right\|}{\left\|\boldsymbol{X}_{k}\right\|}=\ln \left\|\boldsymbol{X}_{k} /\right\| \boldsymbol{X}_{k}\left\|+\sigma \sum_{i=1}^{\mu} w_{i} \boldsymbol{Z}_{i: \lambda}\right\| .
$$

Using the isotropy of the sphere function and of the multivariate normal distribution, together with the previous lemma, we find that the random variables in the RHS of the previous equation are i.i.d. distributed as $\ln \left\|\boldsymbol{e}_{1}+\sigma \sum_{i=1}^{\mu} w_{i} \boldsymbol{Z}_{i: \lambda}\right\|$. Applying the Law of Large Numbers to

$$
\frac{1}{\lambda k} \ln \frac{\left\|\boldsymbol{X}_{k}\right\|}{\left\|\boldsymbol{X}_{0}\right\|}=\frac{1}{\lambda k} \sum_{i=0}^{k-1} \ln \frac{\left\|\boldsymbol{X}_{i+1}\right\|}{\left\|\boldsymbol{X}_{i}\right\|}
$$

we find thus that

$$
\frac{1}{\lambda k} \ln \frac{\left\|\boldsymbol{X}_{k}\right\|}{\left\|\boldsymbol{X}_{0}\right\|}=\frac{1}{\lambda} E\left[\ln \left\|\boldsymbol{e}_{1}+\sum_{i=1}^{\mu} \sigma w_{i} \boldsymbol{Z}_{i: \lambda}\right\|\right]
$$

We develop the convergence in the RHS of the previous equation using the identity

$$
\ln \left\|\boldsymbol{e}_{1}+u\right\|=\frac{1}{2} \ln \left[1+2 u_{1}+\|u\|^{2}\right], \text { for } u \in \mathbb{R}^{n}
$$

that can be obtained in a straightforward way by writing $\ln \left\|\boldsymbol{e}_{1}+u\right\|$ as $\frac{1}{2} \ln \left\|\boldsymbol{e}_{1}+u\right\|^{2}$ and developing the norm. We then obtain that (2) holds with the convergence rate $\mathrm{CR}$ as given in the theorem. To obtain the convergence in expectation as defined in (1), we take the expectation in (5). For a more detailed argumentation why the expectation exists and for the independence of the random variables $\ln \left\|\boldsymbol{X}_{k}\right\| /\left\|\boldsymbol{X}_{k}\right\|$, we refer to [8].

The convergence rate of the $\left(\mu / \mu_{\mathrm{w}}, \lambda\right)$-ES is a function of $\sigma$ and the weights. The optimal convergence rate computes with $\mathrm{CR}(\sigma, \boldsymbol{w})$ from (4) and the constraint $\sum\left|w_{i}\right|=1$ to

$$
\mathrm{CR}_{\left(\mu / \mu_{\mathrm{W}}, \lambda\right)}^{\mathrm{opt}}=\max _{\boldsymbol{y} \in \mathbb{R}^{\mu}} \mathrm{CR}_{\left(\mu / \mu_{\mathrm{W}}, \lambda\right)}\left(\sum_{i=1}^{d}\left|y_{i}\right|, \frac{\boldsymbol{y}}{\sum_{i=1}^{d}\left|y_{i}\right|}\right) \text {. }
$$


Optimal weights can be obtained as $w_{i}^{\mathrm{opt}}=y_{i}^{\mathrm{opt}} / \sum_{i=1}^{\mu}\left|y_{i}^{\mathrm{opt}}\right|$ with $\boldsymbol{y}^{\text {opt }}=\operatorname{argmax}_{\boldsymbol{y} \in \mathbb{R}^{\mu}} \mathrm{CR}_{\left(\mu / \mu_{\mathrm{W}}, \lambda\right)}\left(\sum_{i=1}^{d}\left|y_{i}\right|, \boldsymbol{y} / \sum_{i=1}^{d}\left|y_{i}\right|\right)$ and the optimal step-size equals $\sum_{i=1}^{\mu}\left|y_{i}^{\mathrm{opt}}\right|$.

The convergence rate of the $\left(\mu / \mu_{\mathrm{w}}, \lambda\right)$-ES with scale-invariant constant step-size gives the upper bound for the convergence rate of any step-size adaptive $\left(\mu / \mu_{\mathrm{W}}, \lambda\right)$-ES with isotropic mutations on spherical functions [8, Theorem 1].

\subsubsection{Asymptotic Results}

In the following, we investigate the limit of the convergence rate when the dimension of the search space goes to infinity.

THEOREM 2. The convergence rate of the $\left(\mu / \mu_{\mathrm{w}}, \lambda\right)-E S$ on the class of spherical functions $g(\|x\|), g \in \mathcal{M}$, given scale-invariant step-size and $\sum_{i=1}^{\mu}\left|w_{i}\right|=1$, satisfies

$$
\lim _{d \rightarrow \infty} d \mathrm{CR}\left(\frac{\sigma}{d}, \boldsymbol{w}\right)=-\frac{1}{\lambda}\left(\frac{\sigma^{2}}{2} \sum_{i=1}^{\mu} w_{i}^{2}+\sigma \sum_{i=1}^{\mu} w_{i} E\left(\mathcal{N}_{i: \lambda}\right)\right)
$$

where $\mathcal{N}_{i: \lambda}$ is the ith order statistic of $\lambda$ independent normal distributions with mean 0 and variance 1 , i.e., the ith smallest of $\lambda$ independent variables $\mathcal{N}_{i} \sim N(0,1)$.

Proof. Let $\left(\mathcal{N}^{i}\right)_{1 \leq i \leq \lambda}$ be $\lambda$ independent standard multivariate normal distributions. With the set of all permutations of $\{1, \ldots, \lambda\}$ denoted by $\mathcal{P}(\lambda)$, we have that

$$
\begin{gathered}
\frac{1}{2 \lambda} \ln \left[1+2 \frac{\sigma}{d} \sum_{i=1}^{\mu} w_{i}\left[\boldsymbol{Z}_{1: i}\right]_{1}+\frac{\sigma^{2}}{d} \frac{\left\|\sum_{i=1}^{\mu} w_{i} \boldsymbol{Z}_{1: i}\right\|^{2}}{d}\right]= \\
\frac{1}{2 \lambda} \sum_{\pi \in \mathcal{P}(\lambda)} \ln \left[1+2 \frac{\sigma}{d} \sum_{i=1}^{\mu} w_{i}\left[\mathcal{N}^{\pi(i)}\right]_{1}+\frac{\sigma^{2}}{d} \frac{\left\|\sum_{i=1}^{\mu} w_{i} \mathcal{N}^{\pi(i)}\right\|^{2}}{d}\right] . \\
1_{\left\{h_{\sigma / d}\left(\mathcal{N}^{\pi(1)}\right) \leq \ldots \leq h_{\sigma / d}\left(\mathcal{N}^{\pi(\lambda)}\right)\right\}}
\end{gathered}
$$

For any permutation $\pi \in \mathcal{P}(\lambda)$ and any $1 \leq i \leq \lambda$

$$
h_{\sigma / d}\left(\mathcal{N}^{\pi(i)}\right)=2\left[\mathcal{N}^{\pi(i)}\right]_{1}+\frac{\sigma}{d}\left\|\mathcal{N}^{\pi(i)}\right\|^{2}
$$

such that $\lim _{d \rightarrow \infty} h_{\sigma / d}\left(\mathcal{N}^{\pi(i)}\right)=2\left[\mathcal{N}^{\pi(i)}\right]_{1}+\sigma$. Therefore,

$$
1_{\left\{h_{\sigma / d}\left(\mathcal{N}^{\pi(1)}\right) \leq \ldots \leq h_{\sigma / d}\left(\mathcal{N}^{\pi(\lambda)}\right)\right\}} \underset{d \rightarrow \infty}{\longrightarrow} 1_{\left\{\left[\mathcal{N}^{\pi(1)}\right]_{1} \leq \ldots\left[\mathcal{N}^{\pi(\lambda)}\right]_{1}\right\}} .
$$

In addition, since every component of the vector $\sum_{i=1}^{\mu} w_{i} \mathcal{N}^{\pi(i)}$ follows a standard normal distribution with mean zero and variance $\sum_{i=1}^{\mu} w_{i}^{2}$, we have by the Law of Large Numbers that $\left\|\sum_{i=1}^{\mu} w_{i} \mathcal{N}^{\pi(i)}\right\|^{2} / d$ converges to $\sum_{i=1}^{\mu} w_{i}^{2}$ and thus

$$
\begin{gathered}
\frac{d}{2} \ln \left[1+2 \frac{\sigma}{d} \sum_{i=1}^{\mu} w_{i}\left[\mathcal{N}^{\pi(i)}\right]_{1}+\frac{\sigma^{2}}{d} \frac{\left\|\sum_{i=1}^{\mu} w_{i} \mathcal{N}^{\pi(i)}\right\|^{2}}{d}\right] \\
\underset{d \rightarrow \infty}{\longrightarrow} \sigma \sum_{i=1}^{\mu} w_{i}\left[\mathcal{N}^{\pi(i)}\right]_{1}+\frac{\sigma^{2}}{2} \sum_{i=1}^{\mu} w_{i}^{2} .
\end{gathered}
$$

Injecting the limits from (9) and (10) into (8), we obtain

$$
\begin{array}{r}
\frac{d}{2 \lambda} \ln \left[1+2 \frac{\sigma}{d} \sum_{i=1}^{\mu} w_{i}\left[\boldsymbol{Z}_{1: i}\right]_{1}+\frac{\sigma^{2}}{d} \frac{\left\|\sum_{i=1}^{\mu} w_{i} \boldsymbol{Z}_{1: i}\right\|^{2}}{d}\right] \\
\underset{d \rightarrow \infty}{\longrightarrow} \sum_{\pi \in \mathcal{P}(\lambda)} \frac{1}{\lambda}\left(\sigma \sum_{i=1}^{\mu} w_{i}\left[\mathcal{N}^{\pi(i)}\right]_{1}+\frac{\sigma^{2}}{2} \sum_{i=1}^{\mu} w_{i}^{2}\right) . \\
1_{\left\{\left[\mathcal{N}^{\pi(1)}\right]_{1} \leq \ldots\left[\mathcal{N}^{\pi(\lambda)}\right]_{1}\right\}} .
\end{array}
$$

In the RHS of the previous equation, we recognize the distribution of order statistics of standard normal distributions. Thus,

$$
\begin{array}{r}
\frac{d}{2 \lambda} \ln \left[1+2 \frac{\sigma}{d} \sum_{i=1}^{\mu} w_{i}\left[\boldsymbol{Z}_{1: i}\right]_{1}+\frac{\sigma^{2}}{d} \frac{\left\|\sum_{i=1}^{\mu} w_{i} \boldsymbol{Z}_{1: i}\right\|^{2}}{d}\right] \underset{d \rightarrow \infty}{\longrightarrow} \\
\frac{1}{\lambda}\left(\sigma \sum_{i=1}^{\mu} w_{i} \mathcal{N}^{i: \lambda}+\frac{\sigma^{2}}{2} \sum_{i=1}^{\mu} w_{i}^{2}\right) \cdot
\end{array}
$$

To find the announced result, we need to obtain the limit in expectation. To do so we need to verify that the random variables are uniformly integrable. For this quite technical step we refer to [8].

The asymptotic "classical" progress rate $\varphi$ of the $\left(\mu / \mu_{\mathrm{w}}, \lambda\right)$ ES is derived from an approximation of $1-\left\|\boldsymbol{X}_{k+1}\right\| /\left\|\boldsymbol{X}_{k}\right\|$ and coincides with the limit from the previous theorem

$$
\varphi_{\left(\mu / \mu_{\mathrm{W}}, \lambda\right)}(\sigma, \boldsymbol{w})=\lambda \lim _{d \rightarrow \infty} d \mathrm{CR}\left(\frac{\sigma}{d}, w\right) .
$$

As for the finite dimensional case, we consider the variable $\boldsymbol{y}=\sigma \cdot \boldsymbol{w} \in \mathbb{R}^{\mu}$ with $\sigma=\sum_{i=1}^{d}\left|y_{i}\right|$ and compute the optimal asymptotic convergence rate that is reached for $y_{i}^{\text {opt }}=-E\left(\mathcal{N}_{i: \lambda}\right)$ to

$$
\mathrm{CR}_{\left(\mu / \mu_{\mathrm{W}}, \lambda\right)}^{\mathrm{opt}, \infty}=\max _{\boldsymbol{y} \in \mathbb{R}^{\mu}} \lim _{d \rightarrow \infty} d \mathrm{CR}\left(\frac{\sigma}{d}, \frac{\boldsymbol{y}}{\sigma}\right)=\frac{1}{2 \lambda} \sum_{i=1}^{\mu} E\left(\mathcal{N}_{i: \lambda}\right)^{2}
$$

as already found in [2]. Optimal weights are proportional to $y_{i}^{\text {opt }}$, thus $w_{i}^{\text {opt, } \infty}=-E\left(\mathcal{N}_{i: \lambda}\right) / \sum_{i=1}^{\mu}\left|E\left(\mathcal{N}_{i: \lambda}\right)\right|$. Whether or not negative weights are allowed does not effect the optimal positive weight values, aside from the normalization factor.

\subsection{The $\left(\mu / \mu_{\mathrm{w}}, \lambda\right)$-ES With Random and Selec- tive Mirroring}

Following the same approach as in the previous section, we analyze the convergence rates of the different mirroring variants first for finite dimension and then asymptotically in the dimension. We define as $\left(\boldsymbol{Z}_{1}, \ldots, \boldsymbol{Z}_{\lambda_{\text {iid }}}\right)$, the vector of ordered steps to be recombined; namely for a given ES variant, the best point to be recombined (for which the highest weight will be given) is $\boldsymbol{X}_{k}+\sigma\left\|\boldsymbol{X}_{k}\right\| \boldsymbol{Z}_{1}$, the second best $\boldsymbol{X}_{k}+\sigma\left\|\boldsymbol{X}_{k}\right\| \boldsymbol{Z}_{2}, \ldots$ Among the different algorithm variants, the distribution of the vector of ordered steps changes. In the sequel, we express this distribution for the ES variants with random mirroring, selective mirroring and selective mirroring with resampled length.

Selected vector for random mirroring. In random mirroring, we mirror $\lambda_{\mathrm{m}}$ arbitrary vectors among the $\lambda_{\text {iid }}$ independent ones. Without loss of generality, we can mirror the $\lambda_{\mathrm{m}}$ last vectors. For the mirrored pairs, only the best of the two vectors is recombined. The distribution of the resulting vector of ordered steps is expressed in the following lemma:

LEMMA 2. In the $\left(\mu / \mu_{\mathrm{w}}, \lambda_{\mathrm{iid}}+\lambda_{\mathrm{m}}^{\mathrm{rand}}\right)$-ES with scale-invariant step-size on spherical functions, the distribution of the vector of ordered steps to be recombined is given by

$$
\begin{array}{r}
\left(\boldsymbol{Z}_{1}, \ldots, \boldsymbol{Z}_{\lambda_{\mathrm{iid}}}\right)=\operatorname{argsort}\left\{h_{\sigma}\left(\mathcal{N}^{1}\right), \ldots, h_{\sigma}\left(\mathcal{N}^{\lambda_{\mathrm{iid}}-\lambda_{\mathrm{m}}}\right),\right. \\
\min \left\{h_{\sigma}\left(\mathcal{N}^{\lambda_{\mathrm{iid}}-\lambda_{\mathrm{m}}+1}\right), h_{\sigma}\left(-\mathcal{N}^{\lambda_{\mathrm{iid}}-\lambda_{\mathrm{m}}+1}\right)\right\}, \ldots, \\
\left.\min \left\{h_{\sigma}\left(\mathcal{N}^{\lambda_{\mathrm{iid}}}\right), h_{\sigma}\left(-\mathcal{N}^{\lambda_{\mathrm{iid}}}\right)\right\}\right\}
\end{array}
$$

where $h_{\sigma}(\boldsymbol{x})=2[x]_{1}+\sigma\|x\|^{2}$. 
Proof. Let $\left(\mathcal{N}^{i}\right)_{1 \leq i \leq \lambda}$ be $\lambda$ independent standard multivariate normal distributions. At iteration $k$ starting from $\boldsymbol{X}_{k}$, we rank the individuals $\boldsymbol{X}_{k}+\sigma\left\|\boldsymbol{X}_{k}\right\| \mathcal{N}^{i}$ for $1 \leq i \leq$ $\lambda_{\text {iid }}-\lambda_{\mathrm{m}}$ and the best of the mirrored/unmirrored pairs for the $\lambda_{\mathrm{m}}$ last individuals, i.e., we rank $\left\|\boldsymbol{X}_{k}+\sigma\right\| \boldsymbol{X}_{k}\left\|\mathcal{N}^{i}\right\|$ for $1 \leq i \leq \lambda_{\text {iid }}-\lambda_{\mathrm{m}}$ with $\min \left\{\left\|\boldsymbol{X}_{k}+\sigma\right\| \boldsymbol{X}_{k}\left\|\mathcal{N}^{i}\right\|, \| \boldsymbol{X}_{k}-\right.$ $\left.\sigma\left\|\boldsymbol{X}_{k}\right\| \mathcal{N}^{i} \|\right\}$ for $i=\lambda_{\text {iid }}-\lambda_{\mathrm{m}}+1, \ldots, \lambda_{\text {iid }}$. As in Lemma 1 , we find that the ranking does not change if we normalize by $\left\|\boldsymbol{X}_{k}\right\|$ and if we start from $\boldsymbol{e}_{1}$ such that the distribution is determined by the ranking of $\left\|\boldsymbol{e}_{1}+\sigma \mathcal{N}^{i}\right\|$ for $1 \leq i \leq \lambda_{\text {iid }}-\lambda_{\mathrm{m}}$ and $\min \left\{\left\|\boldsymbol{e}_{1}+\sigma \mathcal{N}^{i}\right\|,\left\|\boldsymbol{e}_{1}-\sigma \mathcal{N}^{i}\right\|\right\}$ for $i=\lambda_{\text {iid }}-\lambda_{\mathrm{m}}+1, \ldots, \lambda_{\text {iid }}$. As in Lemma 1 , we square the terms and develop them to find that the distribution is determined by the ranking according to $h_{\sigma}$ as given in (14).

Selected vector for selective mirroring. In selective mirroring, where we mirror the worse offspring, we need to sort the $\lambda_{\text {iid }}$ offspring to determine which offspring to mirror. Let $\mathcal{Y}$ be defined as

$$
\mathcal{Y}:=\left(\boldsymbol{Y}_{1}, \ldots, \boldsymbol{Y}_{\lambda_{\text {iid }}}\right):=\operatorname{argsort}\left\{h_{\sigma}\left(\mathcal{N}^{1}\right), \ldots, h_{\sigma}\left(\mathcal{N}^{\lambda_{\text {iid }}}\right)\right\}
$$

where $h_{\sigma}(\boldsymbol{x})=2[x]_{1}+\sigma\|x\|^{2}$. Then for the worst $\lambda_{\mathrm{m}}$ vectors of $\mathcal{Y}$, we select the pair-wise best among offspring and mirrored one and we keep the other vectors unchanged:

$$
\begin{aligned}
& \boldsymbol{Y}_{i}^{\text {sel }}=\boldsymbol{Y}_{i}, i=1, \ldots, \lambda_{\text {iid }}-\lambda_{\mathrm{m}} \\
& \boldsymbol{Y}_{i}^{\text {sel }}=\operatorname{argmin}\left\{h_{\sigma}\left(\boldsymbol{Y}_{i}\right), h_{\sigma}\left(-\boldsymbol{Y}_{i}\right)\right\}, \lambda_{\text {iid }}-\lambda_{\mathrm{m}}+1 \leq i \leq \lambda_{\text {iid }}
\end{aligned}
$$

Finally, as expressed in the following lemma, the distribution of the $\lambda_{\text {iid }}$ ordered steps to be recombined is the result of the sorting of the $\boldsymbol{Y}_{i}^{\text {sel }}$ vectors:

LEMmA 3. In the $\left(\mu / \mu_{\mathrm{w}}, \lambda_{\mathrm{iid}}+\lambda_{\mathrm{m}}^{\mathrm{sel}}\right)$-ES with scale-invariant step-size on spherical functions, the distribution of the vector of ordered steps to be recombined is given by

$$
\left(\boldsymbol{Z}_{1}, \ldots, \boldsymbol{Z}_{\lambda_{\text {iid }}}\right)=\operatorname{argsort}\left\{h_{\sigma}\left(\boldsymbol{Y}_{1}^{\text {sel }}\right), \ldots, h_{\sigma}\left(\boldsymbol{Y}_{\lambda_{\text {iid }}}^{\text {sel }}\right)\right\},
$$

where $\boldsymbol{Y}_{i}^{\text {sel }}$ is defined in (15) and (16).

Proof. As in Lemma 1 and Lemma 2, the ranking can be done normalizing by $\boldsymbol{X}_{k}$ and starting from $\boldsymbol{e}_{1}$. Thus, it follows from the way we have defined $\boldsymbol{Y}_{i}^{\text {sel }}$ that the distribution of the vector of ordered steps is determined by (17).

Selected vector for selective mirroring with resampled length. The selective mirroring with resampled length algorithm differs from the previous one for the mirroring step in that only the direction is kept and the length is independently resampled according to its original $\chi$-distribution with $d$ degrees of freedom. Assuming that sorting of the $\lambda_{\text {iid }}$ offspring has been made according to $\mathcal{Y}$ as described above, the $\boldsymbol{Y}^{\text {sel }}$ vector is given by

$$
\boldsymbol{Y}_{i}^{\text {sel }}=\boldsymbol{Y}_{i} \text { for } i=1, \ldots, \lambda_{\mathrm{iid}}-\lambda_{\mathrm{m}}
$$

and for $i=\lambda_{\text {iid }}-\lambda_{\mathrm{m}}+1, \ldots, \lambda_{\mathrm{iid}}$,

$$
\boldsymbol{Y}_{i}^{\text {sel }}=\operatorname{argmin}\left\{h_{\sigma}\left(\boldsymbol{Y}_{i}\right), h_{\sigma}\left(-\left\|\widetilde{\mathcal{N}}^{i}\right\| \frac{\boldsymbol{Y}_{i}}{\left\|\boldsymbol{Y}_{i}\right\|}\right)\right\}
$$

where $\widetilde{\mathcal{\mathcal { N }}^{i}}$ are independent vectors following a standard multivariate normal distribution. As for the previous algorithm, the distribution of the $\lambda_{\text {iid }}$ ordered steps to be recombined is the result of the sorting of the $\boldsymbol{Y}_{i}^{\text {sel }}$ vectors:
Lemma 4. In the $\left(\mu / \mu_{\mathrm{w}}, \lambda_{\mathrm{iid}}+\lambda_{\mathrm{m}}^{\mathrm{sel}}\right)$-ES with scale-invariant step-size on spherical functions, the distribution of the vector of ordered steps to be recombined is given by

$$
\left(\boldsymbol{Z}_{1}, \ldots, \boldsymbol{Z}_{\lambda_{\text {iid }}}\right)=\operatorname{argsort}\left\{h_{\sigma}\left(\boldsymbol{Y}_{1}^{\text {sel }}\right), \ldots, h_{\sigma}\left(\boldsymbol{Y}_{\lambda_{\text {iid }}}^{\text {sel }}\right)\right\},
$$

where $\boldsymbol{Y}_{i}^{\text {sel }}$ is defined in (18) and (19).

Proof. As in Lemma 1 and Lemma 2, the ranking can be done normalizing by $\boldsymbol{X}_{k}$ and starting from $\boldsymbol{e}_{1}$. Thus, it follows from the way we have defined $\boldsymbol{Y}_{i}^{\text {sel }}$ that the distribution of the vector of ordered steps is determined by (20).

Similarly as for the $\left(\mu / \mu_{\mathrm{w}}, \lambda\right)$-ES, we find that the convergence rate of the $\left(\mu / \mu_{\mathrm{w}}, \lambda_{\text {iid }}+\lambda_{\mathrm{m}}^{\text {rand }}\right)$-ES and the $\left(\mu / \mu_{\mathrm{w}}, \lambda_{\text {iid }}+\right.$ $\left.\lambda_{\mathrm{m}}^{\mathrm{sel}}\right)$-ES with and without resampled mutation lengths can be expressed in the following way

THEOREM 3. The convergence rate of the $\left(\mu / \mu_{\mathrm{W}}, \lambda_{\mathrm{iid}}+\right.$ $\left.\lambda_{\mathrm{m}}^{\mathrm{rand}}\right)-E S$ and the $\left(\mu / \mu_{\mathrm{w}}, \lambda_{\mathrm{iid}}+\lambda_{\mathrm{m}}^{\mathrm{sel}}\right)-E S$ with and without resampled mutation lengths equals

$\mathrm{CR}(\sigma, \boldsymbol{w})=-\frac{E \ln \left[1+2 \sum_{i=1}^{\mu} \sigma w_{i}\left[\boldsymbol{Z}_{i}\right]_{1}+\left\|\sum_{i=1}^{\mu} \sigma w_{i} \boldsymbol{Z}_{i}\right\|^{2}\right]}{2\left(\lambda_{\text {iid }}+\lambda_{\mathrm{m}}\right)}$

where $w_{i} \in \mathbb{R}$ and $\sum_{i=1}^{\mu}\left|w_{i}\right|=1$ and the distributions of the random vector $\left(\boldsymbol{Z}_{1}, \ldots, \boldsymbol{Z}_{\lambda_{\text {iid }}}\right)$ are defined in Lemma 2 , 3 and 4 respectively.

Proof. The proof is similar to the proof of Theorem 1 injecting the distribution of the random vectors $\left(\boldsymbol{Z}_{1}, \ldots, \boldsymbol{Z}_{\lambda_{\text {iid }}}\right)$ for the different algorithms.

As for the $\left(\mu / \mu_{\mathrm{w}}, \lambda\right)$-ES, optimal convergence rates are solutions of the maximization problem

$$
\max _{\boldsymbol{y} \in \mathbb{R}^{\mu}} \operatorname{CR}\left(\sum_{i=1}^{d}\left|y_{i}\right|, \frac{\boldsymbol{y}}{\sum_{i=1}^{d}\left|y_{i}\right|}\right)
$$

with CR from Theorem 3.

\subsubsection{Asymptotic Results}

We investigate the limit of the convergence rate given in Theorem 3 when the dimension goes to infinity. For the $\left(\mu / \mu_{\mathrm{W}}, \lambda_{\text {iid }}+\lambda_{\mathrm{m}}^{\mathrm{rand}}\right)$-ES, we define the random vector $\left(Z_{1}, \ldots, Z_{\lambda_{\text {iid }}}\right) \in \mathbb{R}^{\lambda_{\text {iid }}}$ as

$$
\begin{aligned}
& \left(Z_{1}, \ldots, Z_{\lambda_{\text {id }}}\right)=\operatorname{argsort}\left\{\mathcal{N}^{1}, \ldots, \mathcal{N}^{\lambda_{\text {iid }}-\lambda_{\mathrm{m}}},\right. \\
& \left.-\left|\mathcal{N}^{\lambda_{\text {iid }}-\lambda_{\mathrm{m}}+1}\right|, \ldots,-\left|\mathcal{N}^{\lambda_{\mathrm{iid}}}\right|\right\}
\end{aligned}
$$

where the $\mathcal{N}^{i}$ are $\lambda_{\text {iid }}$ independent standard normal distributions. For the $\left(\mu / \mu_{\mathrm{w}}, \lambda_{\mathrm{iid}}+\lambda_{\mathrm{m}}^{\mathrm{sel}}\right)$-ES with or without resampled lengths, we define the vector

$$
\begin{aligned}
\left(Z_{1}, \ldots, Z_{\lambda_{\text {iid }}}\right)=\operatorname{argsort} & \left\{Y_{1}, \ldots, Y_{\lambda_{\text {iid }}-\lambda_{\mathrm{m}}},\right. \\
& \left.-\left|Y_{\lambda_{\text {iid }}-\lambda_{\mathrm{m}}+1}\right|, \ldots,-\left|Y_{\lambda_{\text {iid }}}\right|\right\}
\end{aligned}
$$

where $\left(Y_{1}, \ldots, Y_{\lambda_{\text {iid }}}\right)=\operatorname{argsort}\left\{\mathcal{N}^{1}, \ldots, \mathcal{N}^{\lambda_{\text {iid }}}\right\}$. The asymptotic convergence rate for different variants is given in the following theorem.

THEOREM 4. The convergence rate of the $\left(\mu / \mu_{\mathrm{w}}, \lambda_{\mathrm{iid}}+\right.$ $\left.\lambda_{\mathrm{m}}^{\mathrm{rand}}\right)$-ES and the $\left(\mu / \mu_{\mathrm{w}}, \lambda_{\mathrm{iid}}+\lambda_{\mathrm{m}}^{\mathrm{sel}}\right)-E S$ (with or without resampled lengths) with scale-invariant step-size and weights 

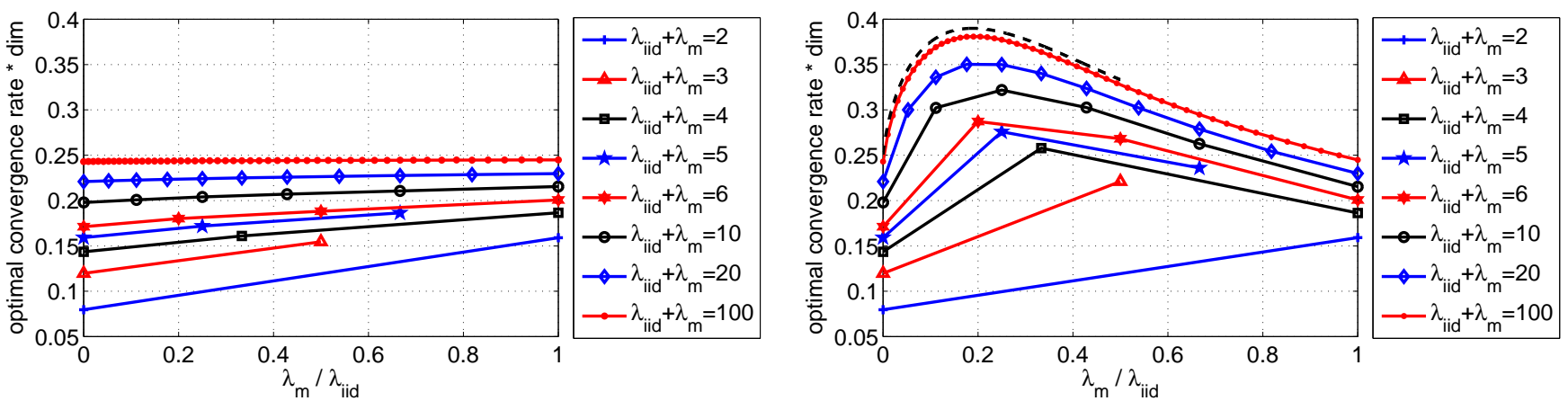

Figure 3: Optimal asymptotic convergence rates $\mathrm{CR}^{\mathrm{opt}, \infty}\left(\lambda_{\text {iid }}, \lambda_{\mathrm{m}}\right)$, Equation $(24)$, for the $\left(\mu / \mu_{\mathrm{w}}, \lambda_{\mathrm{iid}}+\lambda_{\mathrm{m}}\right)-\mathrm{ES}$ versus the ratio $\lambda_{\mathrm{m}} / \lambda_{\text {iid }}$ of mirrored and independent offspring for various $\lambda=\lambda_{\text {iid }}+\lambda_{\mathrm{m}}$. Left: $\left(\mu / \mu_{\mathrm{w}}, \lambda_{\mathrm{iid}}+\lambda_{\mathrm{m}}^{\text {rand }}\right)-$ ES with random mirroring. Right: $\left(\mu / \mu_{\mathrm{w}}, \lambda_{\mathrm{iid}}+\lambda_{\mathrm{m}}^{\mathrm{sel}}\right)$-ES with selective mirroring, mirroring the worst $\lambda_{\mathrm{m}}$ from $\lambda_{\text {iid }}$ independent offspring. In addition, the righthand plot shows the theoretical result for $\lambda_{\text {iid }} \rightarrow \infty$ of Equation (25) as a dashed line.

$\boldsymbol{w} \in \mathbb{R}^{\mu}$ with $\sum_{i=1}^{\mu}\left|w_{i}\right|=1$ on the class of spherical functions $g(\|x\|), g \in \mathcal{M}$ satisfies

$\lim _{d \rightarrow \infty} d \operatorname{CR}\left(\frac{\sigma}{d}, w\right)=\frac{-1}{\lambda_{\mathrm{iid}}+\lambda_{\mathrm{m}}}\left(\frac{\sigma^{2}}{2} \sum_{i=1}^{\mu} w_{i}^{2}+\sigma \sum_{i=1}^{\mu} w_{i} E\left(Z_{i}\right)\right)$

where the distribution of $Z_{i}$ is given in (22) for the $\left(\mu / \mu_{\mathrm{w}}, \lambda_{\mathrm{iid}}+\right.$ $\left.\lambda_{\mathrm{m}}^{\mathrm{rand}}\right)-E S$ and in (23) for the $\left(\mu / \mu_{\mathrm{w}}, \lambda_{\mathrm{iid}}+\lambda_{\mathrm{m}}^{\mathrm{sel}}\right)-E S$ with or without resampled lengths for the mirroring.

Proof. The proof follows the same lines as the proof for the $\left(\mu / \mu_{\mathrm{w}}, \lambda\right)$-ES (Theorem 2 ). The limit is the same for selective mirroring with or without resampled length because asymptotically $\|\mathcal{N}\| /\left\|\mathcal{N}^{\prime}\right\|$ goes to one when $d$ goes to infinity for any two standard multivariate normal distribution $\mathcal{N}$ and $\mathcal{N}^{\prime}$.

Similarly to the $\left(\mu / \mu_{\mathrm{w}}, \lambda\right)$-ES case, we find that the optimal convergence rate is given by

$$
\mathrm{CR}^{\mathrm{opt}, \infty}\left(\lambda_{\mathrm{iid}}, \lambda_{\mathrm{m}}\right)=\frac{1}{2\left(\lambda_{\mathrm{iid}}+\lambda_{\mathrm{m}}\right)} \sum_{i=1}^{\mu} E\left(Z_{i}\right)^{2}
$$

and the optimal weights equal $w_{i}^{\text {opt }}=-E\left(Z_{i}\right) / \sum_{i=1}^{\mu}\left|E\left(Z_{i}\right)\right|$. We remark that the asymptotic convergence rate for the selective mirroring is the same with or without resampled lengths for the mirroring vectors. Thus, the resampling of lengths can only affect finite dimensional results.

We conclude this paragraph with a conjecture on an expression for the optimal asymptotic convergence rate of the $\left(\mu / \mu_{\mathrm{w}}, \lambda_{\text {iid }}+\lambda_{\mathrm{m}}\right)$-ES as a function of $\lambda_{\text {iid }} / \lambda_{\mathrm{m}}$.

CONJECTURE 1. The optimal d-asymptotic convergence rate of the $\left(\mu / \mu_{\mathrm{w}}, \lambda_{\mathrm{iid}}+\lambda_{\mathrm{m}}^{\mathrm{sel}}\right)$-ES with selective mirroring and positive recombination weights in the limit for $\lambda_{\text {iid }} \rightarrow \infty$ and for $r=\lim _{\lambda_{\mathrm{iid}} \rightarrow \infty} \lambda_{\mathrm{m}} / \lambda_{\mathrm{iid}} \leq 1 / 2$ is given by

$$
\begin{aligned}
\mathrm{CR}_{\text {sel mirr }}^{\text {opt }, \infty}(r) & =\frac{1}{2} \frac{1}{1+r}\left(\frac{1}{2}+\int_{-\infty}^{G^{-1}(r)} x^{2} g(x) d x\right) \\
& =\frac{1}{2} \frac{1}{1+r}(\frac{1}{2}+r \underbrace{-G^{-1}(r) g\left(G^{-1}(r)\right)}_{>0 \text { for } 0<r<1 / 2})
\end{aligned}
$$

where $g$ and $G$ are the pdf and cdf of the standard normal distribution respectively.
The $\mathrm{CR}_{\text {sel mirr }}^{\mathrm{opt}, \infty}$ is shown as the top (dashed) graph in Fig. 3, right. $\mathrm{CR}_{\text {sel mirr }}^{\mathrm{opt}, \infty, \infty}$ of 0 and $1 / 2$ compute to $1 / 4$ and $1 / 3$ respectively and its unique maximum in $[0 ; 1 / 2]$ can be found for $\lambda_{\mathrm{m}} / \lambda_{\mathrm{iid}} \rightarrow r=0.188566 \pm 10^{-6}$ as $0.390015661 \pm 10^{-9}$.

\section{SIMULATIONS}

Due to their implicit nature, some of the above derived optimal convergence rates are difficult to compare directly. However, we can easily estimate the rates by means of Monte Carlo sampling allowing us to compare the performance of the proposed algorithms in infinite and finite dimension. Moreover, we find the optimal ratio of mirrored offspring and, theoretically and on spherical functions, the fastest algorithm. All convergence rates are estimated with only positive recombination weights and $10^{6}$ samples are used for each combination of $\lambda_{\text {iid }}$ and $\lambda_{\mathrm{m}}$ and for each algorithm. The MATLAB code is available at http://canadafrance. gforge.inria.fr/mirroring/.

Random and Selective Mirroring in Infinite Dimension. Figure 3 shows estimated optimal convergence rates versus the ratio of mirrored offspring. In all cases, the convergence rate monotonically improves with increasing number of offspring $\lambda$. For random mirroring (left subfigure), the convergence rate also increases monotonically with the number of mirrored offspring $\lambda_{\mathrm{m}}$. The optimal ratio $\lambda_{\mathrm{m}} / \lambda_{\text {iid }}$ is therefore one. With an increasing number of offspring $\lambda$, the convergence rate however approaches 0.25 for any ratio $\lambda_{\mathrm{m}} / \lambda_{\text {iid }}$.

The results look quite different with selective mirroring (right subfigure): for $\lambda_{\mathrm{m}} \in\left\{0, \lambda_{\text {iid }}\right\}$, selective mirroring cannot have any effect, but for any $0<\lambda_{\mathrm{m}}<\lambda_{\text {iid }}$ the convergence rate is consistently better than with random mirroring and has a unique optimum slightly below $\lambda_{\mathrm{m}}=\lambda_{\text {iid }} / 5$.

Figure 4 shows the best convergence rates from Fig. 3 plotted versus $\lambda_{\text {iid }}+\lambda_{\mathrm{m}}$ together with the corresponding optimal ratio $\lambda_{\mathrm{m}} / \lambda_{\text {iid }}$ for selective mirroring. For random mirroring, the known limit convergence rate for $\lambda \rightarrow \infty$ with $\lambda_{\mathrm{m}} \in\left\{0, \lambda_{\text {iid }}\right\}$ is 0.25 (for $\lambda_{\mathrm{m}}=\lambda_{\text {iid }}$ this follows immediately from the optimal value of 0.5 with negative recombination weights). For selective mirroring the limit is close to 0.39 with $\lambda_{\mathrm{m}} / \lambda_{\text {iid }} \approx 0.19$ (see Conjecture 1 above). Note that the unsmoothness of the ratio stems from discretization: not all values for the ratio of $\lambda_{\mathrm{m}} / \lambda_{\text {iid }}$ are possible which in partic- 


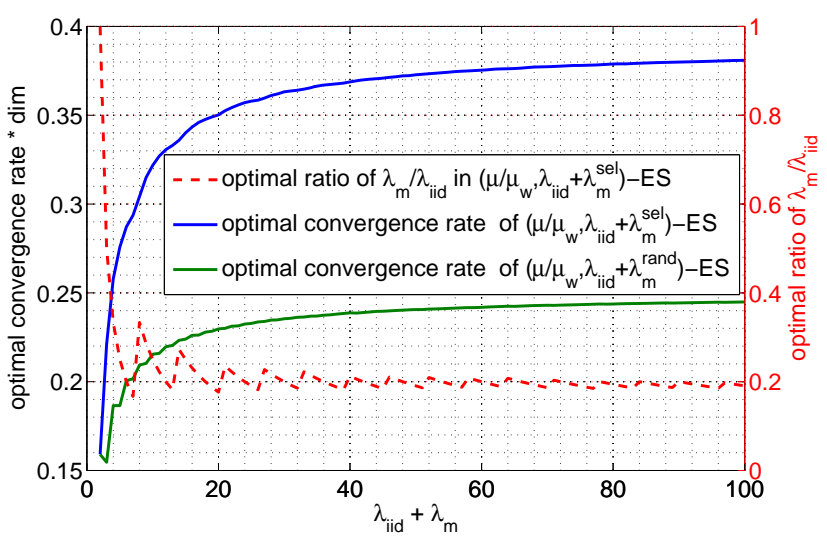

Figure 4: Extracted normalized optimal convergence rates (solid lines) for the $\left(\mu / \mu_{\mathrm{w}}, \lambda_{\mathrm{iid}}+\lambda_{\mathrm{m}}^{\mathrm{rand}}\right)-\mathbf{E S}$ (bottom line) and $\left(\mu / \mu_{\mathrm{w}}, \lambda_{\mathrm{iid}}+\lambda_{\mathrm{m}}^{\mathrm{sel}}\right)$-ES (top line) of Fig. 3 for different numbers of offspring $\lambda=\lambda_{\text {iid }}+\lambda_{\mathrm{m}}$ together with the corresponding optimal ratio of mirrored and unmirrored offspring for the selective mirroring variant (dashed).

ular has an effect for small $\lambda_{\text {iid }}$.

\section{SUMMARY AND CONCLUSION}

We have introduced mirrored sampling in ESs with multirecombination. Two important tricks are used: selective mirroring where only the worst $\lambda_{\mathrm{m}}$ offspring are mirrored and pairwise selection where at most one offspring from any mirrored couple is selected for recombination. Less importantly, the length of mirrored vectors might be resampled. Obtained theoretical results support the effectiveness of selective mirroring in particular: the new algorithm improves the known convergence rate record for ESs with positive recombination weights by $56 \%$ from 0.25 to 0.39 . This is a huge improvement and the new $\left(\mu / \mu_{\mathrm{w}}, \lambda_{\text {iid }}+\lambda_{\mathrm{m}}\right)$-ES, where $\lambda_{\mathrm{m}} \approx 0.19 \lambda_{\text {iid }}$, is also more than $60 \%$ faster than the fastest single-parent mirroring $\left(1+1_{\mathrm{ms}}\right)$-ES and almost twice as fast as the regular $(1+1)-\mathrm{ES}$ in the asymptotic limit, cp. [3].

Only strategies with negative recombination weights are known to realize larger convergence rates, up to 0.5, cp. [2]. Negative weights however have the disadvantage that they use points for recombination that have never been-even remotely - evaluated, that is, they rely on quite specific properties of the fitness function. Compared to the strategy with optimal positive and negative recombination weights, the optimal $\left(\mu / \mu_{\mathrm{w}}, \lambda_{\text {iid }}+\lambda_{\mathrm{m}}\right)$-ES loses out in two ways. About $19 \%$ additional offspring are evaluated - with negative weights they are simply used without being evaluated. These additional evaluations lead to a maximal loss of $1-1 / 1.19=16 \%$ convergence speed. About $0.3 \lambda_{\text {iid }}$ offspring are entirely disregarded for recombination - they have small negative weights otherwise. From the overall loss of $(0.5-0.39) / 0.5=22 \%$ we can imply that the latter disregard contributes with a loss of $6 \%$ in convergence speed.

In preliminary experiments, not shown in this paper, mirrored sampling applied in CMA-ES, using the default recombination weights, improves the convergence speed in small populations, while its effect in large populations is almost negligible ${ }^{6}$. This is not surprising, as with $\lambda \gg d$ mirroring

\footnotetext{
${ }^{6}$ A strong adverse effect that was first observed on a sin-
}

becomes much less effective, because offspring similar to the mirrored ones are already present in the population. Additionally, the reason to apply large populations is not to achieve faster convergence rates $^{7}$.

Considering that small populations are the default setting and that mirrored sampling is simple and has the potential to be cleverly exploited for the covariance matrix update, mirrored sampling might become a future standard method in practice.

\section{REFERENCES}

[1] D.V. Arnold. Optimal weighted recombination. In Foundations of Genetic Algorithms (FOGA 2005), pages 215-237. Springer Verlag, 2005.

[2] D.V. Arnold. Weighted multirecombination evolution strategies. Theoretical computer science, 361(1):18-37, 2006.

[3] A. Auger, D. Brockhoff, and N. Hansen. Analyzing the impact of mirrored sampling and sequential selection in elitist evolution strategies. In Foundations of Genetic Algorithms (FOGA 2011). ACM, 2011. to appear.

[4] A. Auger and N. Hansen. Reconsidering the progress rate theory for evolution strategies in finite dimensions. In Genetic and Evolutionary Computation Conference (GECCO 2006), pages 445-452, 2006.

[5] H.-G. Beyer. The Theory of Evolution Strategies. Natural Computing Series. Springer-Verlag, 2001.

[6] D. Brockhoff, A. Auger, N. Hansen, D. V. Arnold, and T. Hohm. Mirrored sampling and sequential selection for evolution strategies. In Parallel Problem Solving from Nature (PPSN XI), pages 11-21. Springer, 2010.

[7] N. Hansen and A. Ostermeier. Completely Derandomized Self-Adaptation in Evolution Strategies. Evolutionary Computation, 9(2):159-195, 2001.

[8] M. Jebalia and A. Auger. Log-linear convergence of the scale-invariant $\left(\mu / \mu_{w}, \lambda\right)$-ES and optimal $\mu$ for intermediate recombination for large population sizes. Research Report RR-7275, INRIA, june 2010.

[9] A. Ostermeier, A. Gawelczyk, and N. Hansen. Step-size adaption based on non-local use of selection information. In Conference on Problem Solving From Nature (PPSN III), pages 189-198, 1994.

[10] G. Rudolph. Convergence Properties of Evolutionary algorithms. Verlag Dr. Kovac, Hamburg, 1997.

[11] H.-P. Schwefel. Evolution and Optimum Seeking. Sixth-Generation Computer Technology Series. John Wiley \& Sons, Inc., New York, 1995.

[12] O. Teytaud, S. Gelly, and J. Mary. On the ultimate convergence rates for isotropic algorithms and the best choices among various forms of isotropy. In Conference on Parallel Problem Solving from Nature (PPSN IX), pages 32-41. Springer, 2006.

gle multimodal function was due to a too small evaluation budget and vanished under more appropriate experimental conditions.

${ }^{7}$ Note however that the effectiveness of mirroring does not imply that the ES operates only in a local neighborhood predominated by linear terms in the fitness function expansion. 